\title{
Is the Copenhagen Interpretation Compatible with Philosophical Realism?
}

\author{
Leshang Pang ${ }^{1}$ and Dylan Bianchi" ${ }^{1 \#}$ \\ ${ }^{1}$ Auckland International College, Blockhouse Bay, Auckland, New Zealand \\ \#Advisor
}

\section{ABSTRACT}

How are quantum mechanics and realism related? This paper will discuss whether or not the Copenhagen Interpretation of quantum mechanics and the doctrine of philosophical realism are compatible. To answer this question, this paper will first introduce quantum mechanics and the Copenhagen Interpretation in terms of the particle in the box experiment. Then, philosophical realism will be introduced and defined. Finally, the relationship between the Copenhagen Interpretation and philosophical realism will be evaluated. Several points of the Copenhagen Interpretation appear to contradict philosophical realism; thus, it can be concluded that the Copenhagen Interpretation is not compatible with philosophical realism.

\section{Introduction}

It seems to be common sense that the things around us that can be touched, felt, and seen truly exist. One would have subconsciously believed this view of reality ever since they were little. Faye (1) believes that ever since a child, most people have a strong "realistic intuition in the sense that we believe the physical world to exist independently of whether or not it is observed and can be observed" (p. 197). It would not be shocking if someone thought, "the moon is there even if I am not looking at it"; indeed, this is a statement by Albert Einstein which he always believed in. This is the theory of philosophical realism, which was originally developed by the philosopher Aristotle, who is called the father of realism.

It would be unusual and interesting for a realist to think that the moon may be at multiple locations simultaneously until one observes it, but this is the idea of the Copenhagen Interpretation. The Copenhagen Interpretation is the first and the most commonly accepted interpretation of the many theories of quantum mechanics.

Ever since 4900 BCE when the Mesopotamian people first made astronomical observations, classical mechanics was the main and only branch of physics. Classical mechanics aims to describe the motion, behavior, and patterns of macroscopic objects with mathematical formulas. In its modern form, it is built mainly on the laws of Sir Isaac Newton such as the laws of motion and the law of universal gravitation.

This was the heart of physics until the 1920s when quantum theory was developed. Unlike classical mechanics, quantum mechanics studies microscopic objects rather than macroscopic objects and attempts to explain and describe their behavior. The results of these quantum experiments seemed absurd and appeared to contradict certain principles of classical physics. Quantum theory holds that reality and the world around us are probabilistic and that the act of observation can impact the characteristics displayed by objects.

The Copenhagen Interpretation is an interpretation of quantum mechanics. It claims that a quantum particle can have multiple states at once and is only reduced to a definitive state when an observation is made. This might seem to contradict the idea of philosophical realism because the latter considers the world to be determinate and that objects display their characteristics independent of mental human activities. This leads to the question of this paper: Is the Copenhagen Interpretation compatible with philosophical realism? 
This paper will start by identifying the basic ideas of quantum mechanics and the main ideas of the Copenhagen Interpretation and realism that are the most relevant to the discussion. Lastly, the relationship between the Copenhagen Interpretation and philosophical realism will be discussed.

\section{Method}

The main method used is through researching and reading sources to understand the fundamental idea of both Copenhagen Interpretation and philosophical realism. Evaluations and comparisons are then made between the two to understand whether the two are compatible or not.

Before the research process, my hypothesis of the question would be no, Copenhagen Interpretation is not compatible with philosophical Realism. The two appear to be fundamentally different, the former is based on probability and claims that nothing is really certain while the latter argues everything that can be sensed is real.

\section{Quantum Physics}

\section{Quantum mechanics}

One of the pillars of modern physics is quantum mechanics, which studies the microscopic world consisting of things so tiny that they cannot be seen by the naked eye. As discussed above, the results of experiments in quantum mechanics have been very strange and different in contrast to classical physics. Quantum mechanics was built based on other principles that became more accepted between 1900 and 1930. There are many ideas included in the quantum theory, but its main ideas are the following:

- Physical entities have both particle and wave characteristics.

- Quantum mechanics is not deterministic; it is only able to provide probabilities of all possible results.

- Quantum physics applies to the microscopic world.

Things in the world are not particles nor waves, but they display both the properties of waves and the properties of particles. This is what is known as wave-particle duality.

In quantum mechanics, the outcome of a single experiment cannot be predicted and calculated with certainty, but it is always in the form of probabilities. The quantum system can be described mathematically by the wavefunction, which is represented by the symbol psi $(\Psi)$. Though some scholars argue that this wavefunction does exist, others argue that it is just a concept used to describe the state of the quantum system. However, it is agreed that, as the "Born rule" states, the probability of the outcome can be found by taking the square of the wavefunction. The prediction of the location of the particle can only be described in probabilities. Thus, the state of the system before measurements are made cannot be absolutely determined. This characteristic of quantum mechanics led to one of the most famous quotations by Albert Einstein who considered the quantum theory to be incomplete: "God does not play dice."

Quantum mechanics must deal with extremely small particles such as atoms because their small masses and velocities provide large wavelengths that can be observed. As the size of the object increases, the size of the wavelengths decreases. Thus, objects in the macroscopic world have extremely tiny wavelengths that would make their quantum properties difficult to study.

\section{Particle in the Box}

The particle in the box experiment is essential to quantum mechanics. It demonstrates two important characteristics of quantum mechanics: indeterminacy and non-determinism, both of which relate to the probabilistic nature of quantum mechanics.

The particle in the box experiment assumes there to be a very small particle that is placed in a potential energy well or a closed space and it is unable to escape; the particle can move freely within the box. This model is very helpful 
in illustrating the differences between classical physics and quantum physics, as well as the differences between the macroscopic world and the microscopic world.

In classical systems, if a ball is placed in a box with the same velocity at the same angle repeatedly, the ball would be expected to arrive at the same position at the same speed after a fixed amount of time. This can be tested and confirmed by observations. In classical physics, there is no probability regarding the state of objects, their characteristics, properties, and location. It is not more likely for the ball to be found in a certain location than another.

In quantum mechanics, a microscopic particle such as an electron would be used instead of a ball. When the same experiment is repeated multiple times and the position of the electron is measured, it can be observed that it is more likely for the electron to be at one position than another.

If the experiment is repeated and the number of times the electron was found in a specific region is recorded, the result of the repeated experiments provides the probability of where the electron could be. There are places where it is more likely to be and places where there is a smaller or zero chance. These data can be then graphed to form a histogram such as the one diagram below (Figure 1) which shows an example of when the particle in the box system is in its lowest energy state. The $\mathrm{x}$-axis shows the position of the electron by discrete region in which the particle might be found. The y-axis shows the probability of the electron being in that region. Each category of the histogram is the range of areas that the electron can be found in.

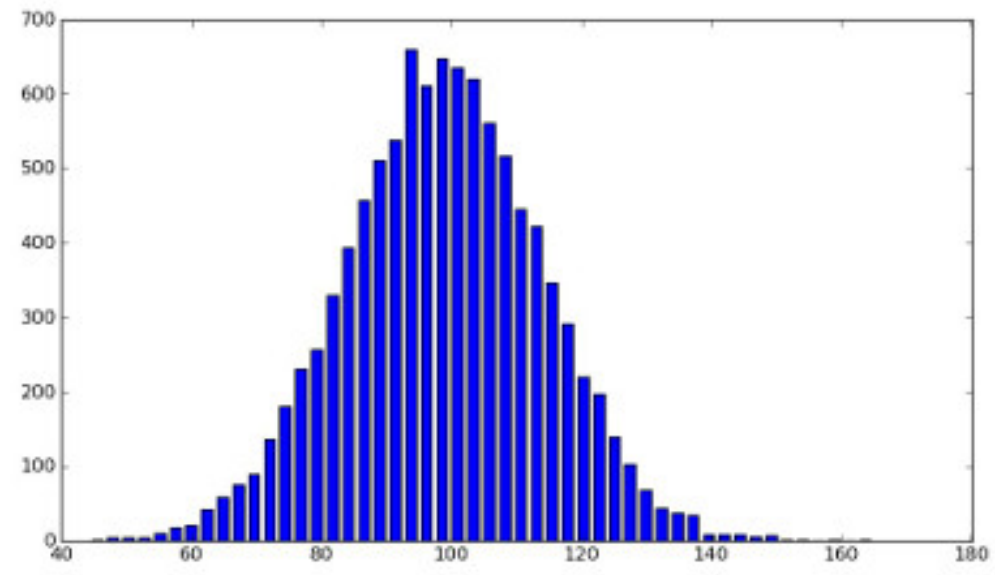

Figure 1: Particle in the box experiment histogram

This histogram approximates the square of the wavefunction, which is the probability density of finding an electron in different regions within the box. If a line is drawn connecting the top of the bars, the shape of that line would approximately reflect the shape of the wavefunction squared.

It is clear from this experiment that the position of the electron is probabilistic. It is also observed that the position is not random because after repeating the experiment, the positions of the electron are at certain locations with certain probabilities are repeated. This shows that there is a higher probability of the electron being at one location than at another location with lower or even zero probability. The electron is free to move, and it moves in a way that cannot be determined because an electron does not have a definite path or location. Thus, the precise position of an electron cannot be calculated at a specific time, but the probability can be calculated.

This experiment illustrates the indeterminacy of the microscopic world according to quantum mechanics. It demonstrates that the world is probabilistic rather than definite. This is one of the main differences between quantum mechanics and classical physics. The particle in the box experiment also shows the non-determinism of the world. As previously mentioned, the path and location of the electron cannot be predicted unlike that of a ball in the classical world. Not being able to predict the future based on the past is another respect in which quantum mechanics differs from classical physics.

\section{Copenhagen Interpretation}




\section{Historical Background}

The Copenhagen Interpretation was formulated by a group of quantum physicists connected with Niels Bohr's Copenhagen Institute during the 1920s. Neither Niels Bohr nor Werner Heisenberg has used the term "Copenhagen Interpretation" as a proper name of their theory. The interpretation was first referred to as "the Copenhagen spirit of quantum theory" in Heisenberg's 1930 book The Physical Principles of the Quantum Theory (2). Since this interpretation was the first, a specific name was not needed until the rise of other interpretations such as the hidden-variable approach and the many-worlds theory.

\section{Copenhagen Interpretation}

Quantum mechanics was a new concept introduced that was not like classical physics, which is based on Newton's laws of motion and gravity. Classical physics treats particles and waves as two separate and completely different matters; it also relies on consistency, determinism, and causality. On the other hand, quantum physics suggests the idea of probability and discontinuity. The Copenhagen Interpretation attempts to articulate the correct way of considering the physical meaning of quantum mechanics along with its mathematical formulations and experimental results, and it is a way to view the relationship between the wavefunction, experimental results, and the nature of reality.

According to the Copenhagen Interpretation, the macroscopic and microscopic worlds are two different realms. The macroscopic world includes objects that can be felt, seen, and measured. This is the classical realm governed by classical physics. The microscopic refers to the small particles that cannot be felt, seen, measured, and make up all matter. The dynamics of these particles are governed by the Schrödinger equation.

The Copenhagen Interpretation is committed to the idea that microscopic physical systems generally do not have definite properties before the measurements are made. The act of measurement causes the wavefunction to collapse; in other words, the probability of all the possible values is reduced to only one immediately after making the measurement. As physicist Brian Greene (3) puts it, "whenever you try to see a probability wave, the very act of observation thwarts your attempt" (p.233). With the particle in the box experiment as an example, there is a range of areas where the electron could be found. The area under the square of the wavefunction, or the height of the bar of the histogram, shows the probability of finding the electron in a given region. However, when observations are made, the electron is only found at one specific location. This fact constitutes the collapse of the wavefunction. The wavefunction changes from a curved graph into a narrow spike which reflects the fact that the electron can only be found at one location after observations have been made.

The Copenhagen Interpretation is interpreted, explained, and presented differently by different authors, writers, and scientists. It is known that even the two main founders of this interpretation, Niels Bohr and Werner Heisenberg, did not agree on many issues. Some scholars suggest that Bohr was influenced by positivism or pragmatism whereas Heisenberg leaned more towards realism. Professor John G. Cramer (4) concludes that "nowhere does there seems to be any concise statement which defines Copenhagen Interpretation" (p. 649).

Despite small variations, eight main ideas are generally accepted as a part of the Copenhagen Interpretation. They are:

- The wavefunction can be used to represent the state of the system.

- Heisenberg's Uncertainty Principle states that there is a limit on the degree of precision to which certain pairs of measurements can be made.

- $\quad$ During the observation, there must be certain interactions between the system and a laboratory device.

- The outcomes of measurements are classical and should be described in ordinary language.

- The description of the wavefunction is probabilistic.

- According to Niels Bohr's complementarity concept, there is a relation between the collapse of the wavefunction and the act of measurement.

- The inner workings of atomic and subatomic processes cannot be known when they are being directly observed because they are impacted by the act of observation. 
- Bohr and Heisenberg's correspondence principle states that the properties of a system become more similar to the classical descriptions as the size of the quantum numbers increase.

\section{Main Claims of the Copenhagen Interpretation}

The focus of this paper is the relationship between the Copenhagen Interpretation and philosophical realism, so our attention will be restricted to the following three claims:

- The state of the system can be represented by the wavefunction.

- There must be interactions between the system and a laboratory device during the observation, and the inner workings of atomic processes cannot be known when they are being directly observed.

- There is a relationship between the collapse of the wavefunction and the act of measurement.

A wavefunction describes the probability of the isolated quantum state of a particle as a function of position, momentum, time, and/or spin. For example, the area under the square of a wavefunction describing a region of space gives the probability of the electron being within this region. When the wavefunction is the complete characterization of the state of the system, this makes the system probabilistic. The square of the wavefunction would provide the probability of the system being at one certain state and the probability of it being at another. Copenhagen Interpretation suggests that the world described by the quantum theory is a world that is vague rather than precise. This world has different properties and does not provide a precise location, momentum, and energy.

To show the probabilistic nature of the microscopic realm, the particle in the box experiment needs to be repeated several times and each time, the position of the electron needs to be recorded. As previously explained, the wavefunction can describe the results of the particle in the box experiment. The area under the wavefunction is the probability of the location of the electron, just like how the area of the box of the histogram shows the chance of the election being between two specific points.

The Copenhagen Interpretation argues that there is a relationship between the collapse of the wavefunction and the act of measurement. When the measurement is made, all the possibilities are reduced to just one. This is the collapse of the wavefunction. The shape of the wavefunction was originally a curve; during the act of observation, the wavefunction reduces to a narrow spike. After the measurement, the wavefunction begins evolving again and slowly returns to its original shape.

The Copenhagen Interpretation states that there must be certain interactions between the system and a laboratory device and that the act of observation can impact the system. This would be quite different from the classical world where the physical properties or characteristics of an object are generally not influenced by the act of measurement. Copenhagen Interpretation, by contrast, holds that the act of observation can change the physical properties of an object.

For the particle in the box experiment, there must be an interaction between the electron, which is part of the system, and a laboratory device such as a photon generator. The photon is bounced off of the electron so that the position and momentum of the electron can be determined. However, this process has certain impacts on the electron, which affect the properties of the electron, so the exact position or the exact momentum of the electron before the measurement was made cannot be known. This also demonstrates the indeterminacy of quantum physics. When measuring the position of the electron, the location of the electron before the observation can never be known because the act of observation causes the collapse of the wavefunction and thus changes the physical property of the electron.

The atoms and electrons that quantum mechanics studies do not seem to follow the principle of classical mechanics, which considers the world to be definite and deterministic. However, in quantum mechanics, things such as the location of the particle seem to be probabilistic. There is a certain chance of it being at point A and another chance of it being at point B. Quantum mechanics considers reality to display both characteristics of indeterminacy and nondeterminism. Indeterminacy is the state of being not definite. With the example of an electron, according to quantum mechanics, it does not have a definite location, momentum, or velocity. Non-determinism is when the future cannot be predicted based on the past. In classical physics, the path and location of a ball can be predicted accurately with 
calculations. This is not the case in quantum mechanics. With the particle in the box experiment as an example, the location and path of the electron cannot be predicted, only a probability of where the electron can be calculated. This would be the main idea of how the Copenhagen Interpretation interprets quantum mechanics.

\section{Realism}

\section{Philosophical Realism}

Philosophical realism holds that the independent existence of objects, and these objects displaying specific characteristics, is not affected by one's view, perception, or ideas. Ted Sider (5) suggests that the world exists independently of the human mind, the world is there to be discovered and there is nothing that can be done to change the world. This is what Sider calls "knee-jerk realism," the general picture that the main purpose of human inquiry is to "conform itself to the world" rather than to create or construct the world (Sider, 2011, p.18).

There are two aspects of philosophical realism. The first aspect concerns the determinate existence of objects and the definiteness of their characteristics. An example would be that the moon exists, is spherical, and orbits around the Earth. This describes the existence and characteristics of the Moon.

The second aspect of philosophical realism is the independent existence of objects. Objects exist and display their traits independently of the mental activities of consciousness. Continuing with the example of the moon, the moon exists, stays spherical, and orbits the Earth regardless of what one thinks of it. If someone thinks the moon is flat or wants the moon to become flat, the moon will still stay spherical because the human mind cannot change the characteristics of an independently existing object.

A realist believes that the characteristics of an object are determined; they do not change due to internal forces nor are they probabilistic. Realism is the general way one would perceive the world. A realist would hold that reality comprises the things that can be seen, felt, sensed, and known; the world is definite, and it cannot be changed by the human mind.

\section{Scientific Realism}

Scientific realism is the belief that scientific theories are true or aimed towards the truth, and they can accurately describe reality. There are several different ways to define scientific realism and different theorists define it differently. Hilary Putnam (6) considers a realist to be someone who "holds that 1 . The sentences of that theory are true or false; and 2. That what makes them true or false is something external - that is to say, it is not (in general) our sense data, actual or potential, or the structure of our minds, or our language, etc)" (p.69). According to Bas van Fraassen (7), scientific realism states that "science aims to give us, in its theories, a true story of what the world is like; and acceptance of a scientific theory involves the belief that it is true" (p. 1066)

On the other hand, a scientific antirealist would think that scientific theories are just useful tools for completing certain calculations or for other purposes, but the theory itself might not be literally true. For example, the model of the atom that is taught in high school is not what an actual atom would look like. However, this helps students to better understand the behavior of atoms, so it is commonly used.

\section{The Relationship between the Copenhagen Interpretation and Philosophical Realism}

Although one can be both a philosophical realist and a scientific antirealist and vice versa, if one is a scientific antirealist then there is no point in discussing the incompatibility between philosophical realism and the Copenhagen 
Interpretation of quantum mechanics. From the scientific antirealist's point of view, theories or concepts such as the Schrödinger Equation can be used to make accurate predictions and calculations, but they do not need to believe that the theory is a true description of the world. If the meaning of quantum theory is irrelevant or insignificant, then there is no need to consider how the Copenhagen Interpretation is related to philosophical realism in the view of a scientific antirealist. The incompatibility of philosophical realism and the Copenhagen Interpretation would only be of interest through the eyes of a scientific realist.

The main points of incompatibility are the act of observation and the probabilistic nature that quantum mechanics describe objects to have. These will be discussed in turn.

\section{The probabilistic nature of objects}

The Copenhagen Interpretation or even quantum mechanics in general states that the world is probabilistic. Everything is indeterminate and non-deterministic. The probabilistic nature of the microscopic realm can be shown with the wavefunction and illustrated through the particle in the box experiment.

This probabilistic feature contradicts philosophical realism, which believes that the world is determined. Philosophical realism, and especially "knee-jerk realism," argues that the world is already "out there," and it cannot be changed or especially changed by humans; it is there for us to explore and find out more about it. Philosophical realism also advocates the idea that reality can be definitively known through scientific inquiry. Thus, it can be concluded that the Copenhagen Interpretation and Philosophical realism are incompatible regarding the question of whether the world is indefinite or probabilistic.

\section{The act of mental observation causing the collapse of the wavefunction}

The act of measurement is the act of consciousness making observations on the object. I have described above how observations cause the wavefunction to collapse.

This phenomenon contradicts philosophical realism. Philosophical realism suggests that human activities or consciousness such as the act of observation would not have any influence on the object's characteristics: the moon is spherical and doing whatever it is doing whether one is looking at it or not. However, this claim is saying that before observations are made, the state of the moon is probabilistic: the moon may not be spherical, and it could be anywhere within a range of locations. The act of observation forces the moon to become spherical and show certain characteristics that are then seen. If it is the physical impact of the act observation that makes the moon spherical, this is not a problem for philosophical realists who must acknowledge that humans do have the power to impact the characteristics of an object through physical intervention. But if the state of the object is influenced by the mental aspect of mental observation, then this would contradict with philosophical realism. It is stated as one of the main aspects of philosophical realism that the characteristics of an object cannot be directly changed by one's idea, thoughts, or theory; this would also include that it cannot be changed by the simple mental act of observation. Thus the main issue here is whether or not this act of observation is mental or physical.

\section{Conclusion}

There are three main claims of the Copenhagen Interpretation that seem to be in tension with philosophical realism: (1) the state of the system can be represented by a wavefunction; (2) the wavefunction provides a series of probabilities rather than determinate characteristics, and (3) the act of observation can change or determine the system's characteristics. Mental observation being able to impact the system is problematic from the perspective of realism because philosophical realism would not agree that consciousness can make changes to other physical objects or systems directly. However, if the observation is a physical intervention, this would be acceptable for a philosophical realist because they agree that human acts can have physical impacts on objects. 


\section{Conclusion}

The question of this paper is whether or not the Copenhagen Interpretation is compatible with philosophical realism. This paper first looked into quantum mechanics, the Copenhagen Interpretation, realism, and lastly discussed the relationship between the Copenhagen Interpretation and realism.

The main contradictions between the Copenhagen Interpretation and philosophical realism are the world being probabilistic rather than determinate and mental observations having an influence on physical objects. A philosophical realist sees the world as determined; the world is already out there for us to discover. However, according to the Copenhagen Interpretation, the world is probabilistic, things such as the location of an electron can be described using a wavefunction and there is a higher chance of the electron being at one certain place than another as described by the particle in the box experiment. A philosophical realist would also consider that objects exist and display their characteristics independently of mental human activities. On the other hand, the Copenhagen Interpretation claims that the act of mental observation can cause the wavefunction to collapse, whereby all the possible locations of the electron are reduced to only one immediately after observation by consciousness has been made.

To answer the question of this paper, the Copenhagen Interpretation is compatible with some parts of philosophical realism. Both sides would agree that objects exist and display their characteristics; however, a believer of the Copenhagen Interpretation would not agree with the philosophical realist's argument about the objects showing their traits independently regardless of all human activities. The Copenhagen Interpretation is also not compatible with philosophical realism about whether the world is probabilistic or determinate. Thus, it can be concluded that overall, the Copenhagen Interpretation is incompatible with philosophical realism.

\section{Acknowledgment}

I would like to express my special thanks to my advisor, Dylan Bianchi for his continuous advice, support, and dedication. I would also like to thank Ms. Zhang and Ms. Li for motivating me and helping me to gain this opportunity. Finally, I would like to thank my friends and family for their mental support and encouragements.

\section{References}

(1) Faye, J. Niels Bohr: His heritage and legacy: An anti-realist view of quantum mechanics. Science and philosophy: 6, Dordrecht, Boston, Kluwer Academic, 1991.

(2) Heisenberg, W. The physical principles of the quantum theory. New York, Dover Publications, 1949.

(3) Greene, B. The hidden reality: Parallel universes and the deep laws of the cosmos. New York, Vintage Books, 2013

(4) Cramer, JG. The transactional interpretation of quantum mechanics. Reviews of Modern Physics, 58, 647-687, 1986.

(5) Sider, T. Writing the Book of the World. Oxford University Press, Oxford, 2011.

(6) Putnam, H. Mathematics, matter, and method. London, New York, Cambridge University Press, 1975.

(7) Van Fraassen, B. Arguments concerning scientific realism. In Martin Curd \& Jan Cover (eds.), Philosophy of Science: The Central Issues. Norton, 1998. 